\title{
OVERLAP CORRECTION FUNCTION FOR AN AIRBORNE BASED LIDAR
}

\author{
Mariana Adam ${ }^{1 *}$, Franco Marenco ${ }^{2}$ \\ ${ }^{1}$ Observations branch, Met Office, Exeter, Devon EX1 3PB, United Kingdom, *Email: \\ mariana.adam@metoffice.gov.uk \\ ${ }^{2}$ Observations Based Research, Met Office, Exeter, Devon EX1 3PB, United Kingdom
}

\begin{abstract}
The present research envisages the estimation of the overlap correction function for an airborne nadir-mounted lidar using multi-angle measurements. We have scanned a series of offnadir angles and after data processing we have been able to determine the instrument's overlap function down to $95 \mathrm{~m}$ from the lidar. This function can be used for the correction of lidar profiles and hence reduce the near-range uncertainty of lidar measurements. To our knowledge, the estimation of the overlap function using multi-angle method for a nadir pointing lidar is a première.
\end{abstract}

\section{INTRODUCTION}

The Met Office is one of the main users of the FAAM BAe-146 research aircraft, alongside scientists working in universities funded by the Natural Environment Research Council [1], and is responsible for maintaining the on-board elastic backscatter lidar. Moreover, as a response to the eruption of Eyjafjallajökull in 2010, the Met Office has set up a second aircraft, the Met Office Civil Contingencies Aircraft (MOCCA), a Cessna 421 which is aimed at giving a rapid response in case of natural hazards (especially volcanic ash) [2].

A Leosphere ALS450 UV Lidar (with depolarization capability) is set up onboard both aircrafts [3]. Besides Lidar, several instruments perform in-situ measurements to characterize the microphysical and optical properties of the aerosol particles and clouds, atmospheric turbulence and gas chemistry [1-2].

The current research evaluates the overlap function of the lidar on-board the FAAM aircraft, using multi-angle measurements, which is based on the method developed by Adam et al. [4] for ground-based lidars.

\section{METHODOLOGY}

For ground-based Lidar, the multi-angle methods are based on measurements taken by scanning several elevation angles; the main assumption being that the atmosphere is horizontally homogeneous. This assumption is most probably fulfilled for airborne based lidars, provided a suitable altitude is selected, as we expect more homogeneity above the boundary layer (very close to a molecular atmosphere). The main steps of the method are the following. For each height, the logarithm of range corrected signals (RCS) (expressed as a function of height) is plotted versus $1 / \cos (\varphi)$, where $\varphi$ the off-nadir pointing angle. The slope of the linear regression provides the optical depth. Once we retrieve the optical depth $\tau(0, h)$ and the intercept $A^{*}(h)$ from the linear regression, a synthetic signal for $R C S$ is calculated as in [4]:

$$
Z_{j}^{*}(h)=\exp \left[A^{*}(h)\right] \exp \left[-2 \tau(0, h) / \cos \varphi_{j}\right](1)
$$

where $j$ is the angle index. The individual overlap function is then computed as the ratio of the measured $R C S$ and synthetic $R C S$ as a function of height $h$.

$q_{j}(h)=P_{j}(h)\left(h / \cos \varphi_{j}\right)^{2} / Z_{j}^{*}(h)$

The final steps are the conversion of individual overlap function (eq. 2) in terms of range and then compute the average overlap function [4].

The method was first tested over synthetic data. Sensitivity studies were employed to find out a comfortable set of bank angles capable of yielding to a good estimate of the overlap function. The main constraint is given by the capability of the aircraft to provide specific measurements at high bank angles. The Lidar is installed with a fixed pointing angle with respect to the aircraft and thus, we need to roll the aircraft in a turn in order to change the bank angle. As the aircraft spins around 360 degrees with a special bank angle we have an orbit. The steepest orbit available with the 
BAe-146 aircraft is one with a 60 degrees bank angle, and it impresses on cabin occupants an acceleration of $2 \mathrm{~g}$. The aircraft pitch and roll are recorded every second by the on-board navigation system, and can thus be easily linked to the lidar data.

\section{EXPERIMENT}

The first flight performing orbits according to the scientific needs for the overlap study, took place on $17^{\text {th }}$ of June 2014 (as part of a broader mission, along with other scientific objectives). The orbits were performed on the nominal bank angles: $30^{\circ}$, $40^{\circ}, 50^{\circ}$ and $60^{\circ}$. The Lidar did not perform optimally during this flight (laser energy was low and thus SNR was low). The cruise altitude was $\sim 3.9 \mathrm{~km}$. However, the method was tested and the result was promising even if it was relatively noisy. Thus, during a subsequent flight, on $10^{\text {th }}$ of September 2014, the specific flying strategy for the overlap study was repeated. In addition to the initial bank angles, the $10^{\circ}$ and $20^{\circ}$ bank angles were added. However, the cruise altitude was different $(\sim 4.8 \mathrm{~km})$. The flight took place over the Ocean, near the coast of Cornwall. Note that the $30^{\circ}, 40^{\circ}$ and $60^{\circ}$ orbit was repeated twice in order to increase the number of available data points (a $60^{\circ}$ orbit lasts less than 1 minute). The data quality was superior and thus we chose this set of data for final analyses. The flight track is shown in Fig. 1. In addition to the nine orbits, two runs were performed at $\sim 0^{\circ}$ and we consider these data as well.

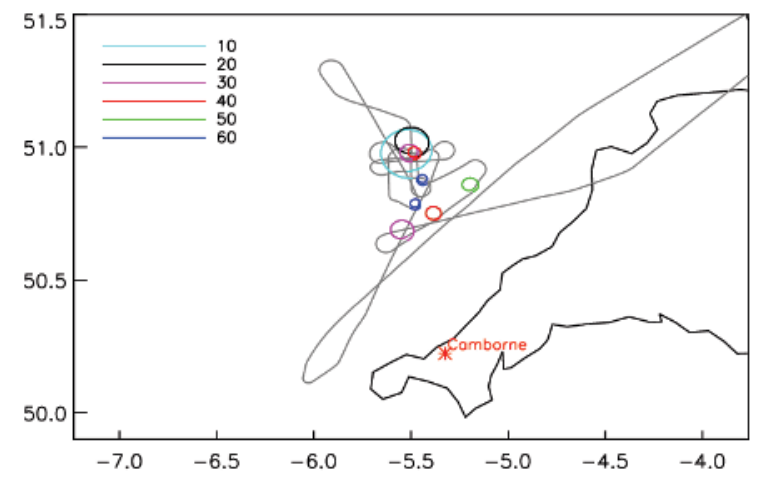

Fig. 1. Flight track.

The measurements are taken with a range resolution of $1.5 \mathrm{~m}$ and an integration time of $2 \mathrm{~s}$. In the first stage, the number of measurements for each nominal bank angle was calculated, according to the flight summary. The cruise altitudes and the bank angles for the entire flight are shown in Fig. 2.

Figure 3 shows the bank angles used in the overlap experiment. As seen in both figures, in reality, there is a slight deviation from the nominal bank angles. The mean altitudes (along with STD) corresponding to the mean bank angles are shown in Table 1 and Fig. 3. The number of measurements for each bank angle is shown in the third column in Table 1. The overall mean cruise altitude is $4837.6 \mathrm{~km}$ while the STD is $13.76 \mathrm{~m}$.

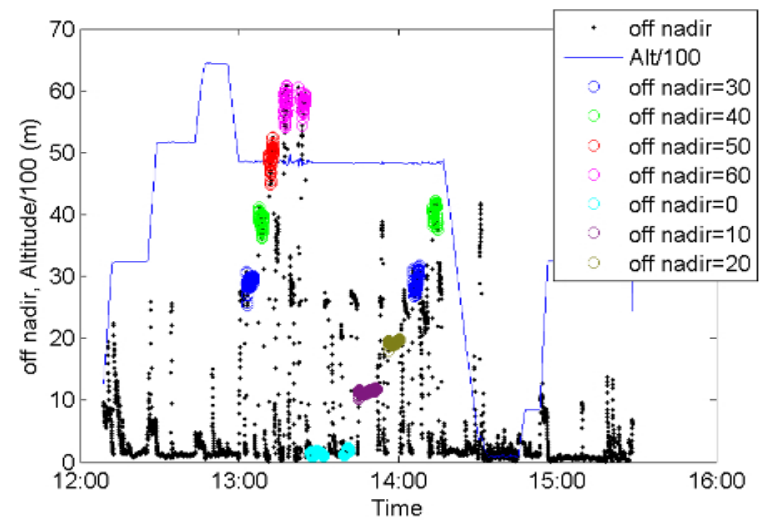

Fig. 2. Cruise altitudes and bank angles (off-nadir) for entire flight.

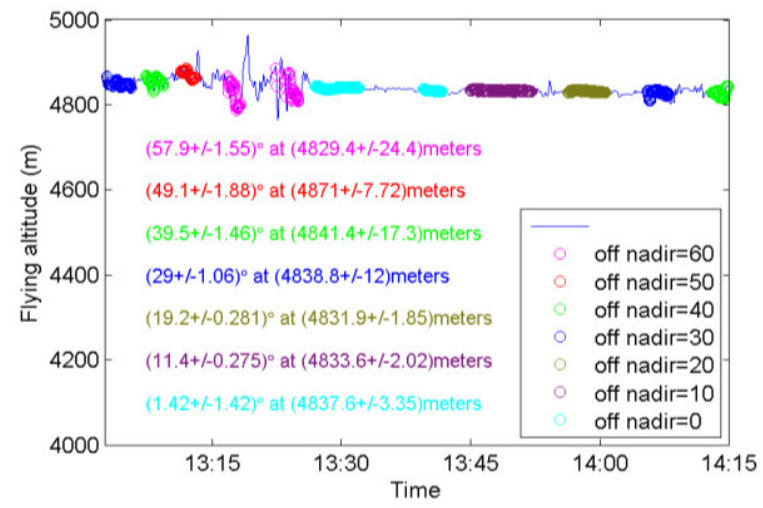

Fig. 3. The cruise altitudes for all the orbits performed for the planned bank angles (off-nadir) of $10^{\circ}, 20^{\circ}, 30^{\circ}, 40^{\circ}, 50^{\circ}$ and $60^{\circ}$ and runs at $0^{\circ}$. The mean bank angle and the corresponding mean altitude are shown.

Table 1. Mean bank angle and altitude cruise

\begin{tabular}{|l|l|l|}
\hline Mean bank angle & Mean cruise altitude & \# points \\
\hline $11.4 \pm 0.275$ & $4833.6 \pm 2.02$ & 203 \\
\hline $19.2 \pm 0.281$ & $4831.9 \pm 1.85$ & 123 \\
\hline
\end{tabular}




\begin{tabular}{|l|l|l|}
\hline $29 \pm 1.06$ & $4838.8 \pm 12$ & 153 \\
\hline $39.5 \pm 1.46$ & $4841.4 \pm 17.3$ & 110 \\
\hline $49.1 \pm 1.88$ & $4871 \pm 7.72$ & 48 \\
\hline $57.9 \pm 1.55$ & $4829.4 \pm 24.4$ & 79 \\
\hline
\end{tabular}

\section{RESULTS}

According to the lidar specifications, the full overlap should be around $300 \mathrm{~m}$. Time-altitude $R C S$ profiles for the total signal (as a combination of parallel and perpendicular signals) is shown in Fig. 4 a). PBL height was $\sim 1 \mathrm{~km}$ at Camborne and $\sim 1.3 \mathrm{~km}$ at Exeter (as seen in ceilometers). While at Camborne it was cloud free atmosphere, at Exeter there were clouds in the PBL. As seen in Fig. $4 \mathrm{~b}$, there is an increase in the RCS below $1.5 \mathrm{~km}$ which may be due to the presence of a cloud in the PBL. However, the region below $1.5 \mathrm{~km}$ altitude will not affect the overlap retrieval (which is of interest in the first few hundred meters from the aircraft which is situated more than $2000 \mathrm{~m}$ above the PBL). For each bank angle, the mean (total) RCS is calculated [Fig. 4 b)].
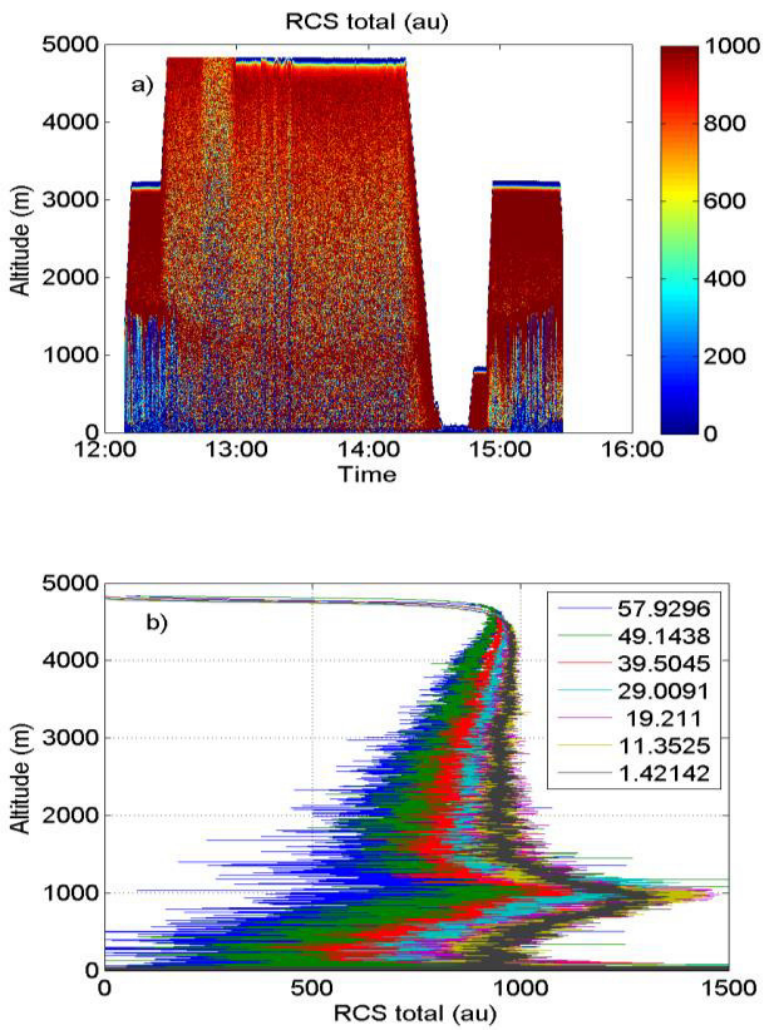

Fig. 4. Total $R C S$ over the entire flight (a). Mean (total) $R C S$ for each bank angle (b).
The flight took place about $\sim 100 \mathrm{~km}$ north of Camborne site (Fig. 1). In the paper by Adam [4], for zenith pointing angles Lidar, there are few criteria involved such that we use the correct altitude interval to perform the linear regression ("optimal heights interval") without introducing systematic errors. Those criteria should be adapted for nadir pointing Lidar as the signals look different as compared with zenith pointing lidars. As described in [4], the use of data from incomplete overlap introduces systematic distortions in the retrieval the intercept (and further in the overlap). In the first step we did not restrict the region over the incomplete overlap and thus, all the measurements were taken into consideration. The optical depth and the intercept retrieved in this case are shown in Fig. 5 (down to $1500 \mathrm{~m}$ altitude). Also shown, the molecular optical depth, which is calculated using the pressure and temperature profile as given by the radiosonde launched at 12:00 at Camborne. As seen, the optical depth obtained over the incomplete overlap is negative (from the cruise altitude down to $\sim 4500 \mathrm{~m}$ ).
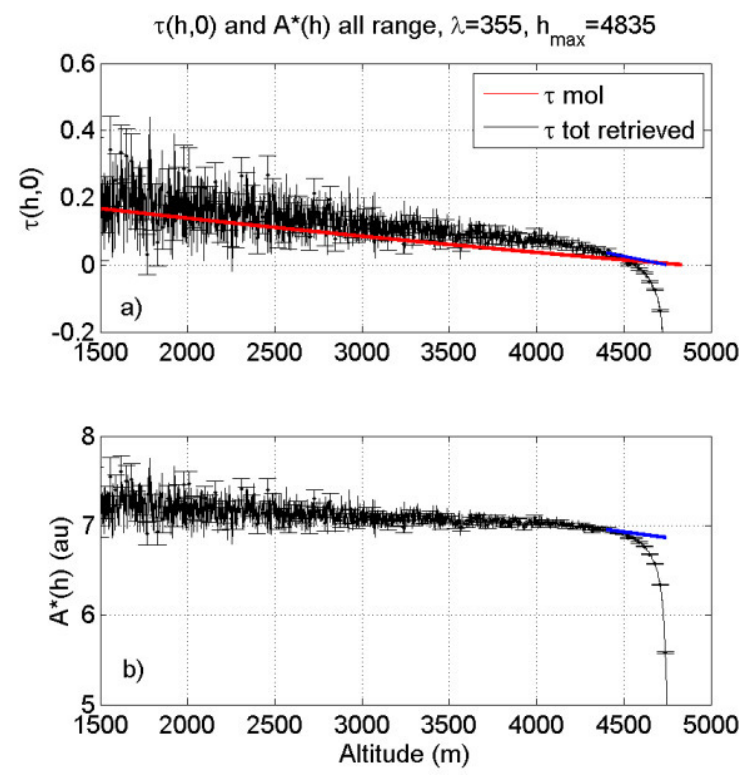

Fig. 5. Optical depth (a) and the intercept (b).

In order to avoid the use of data from incomplete overlap, we have chosen to the following option. We extrapolated the optical depth and the intercept from $4400 \mathrm{~m}$ towards the Lidar using a linear fit over the region $4200 \mathrm{~m}-4400 \mathrm{~m}$. The extrapolation is shown as blue line in Fig. 5. Further we have dismissed the first $95 \mathrm{~m}$ from the 
Lidar. Now we proceed to compute the synthetic $R C S$ [eq. (1)] and the individual overlap functions [eq. (2)]. The overlap function, calculated as the average of the individual functions, is shown in Fig. 6. The complete overlap is shown to be around $400 \mathrm{~m}$ (it is $\sim 0.98$ at $300 \mathrm{~m}$, the range of the nominal full overlap according to specifications). The overlap function looks very good in the far field up to $5000 \mathrm{~m}$. Note that the errors were computed using the error propagation with input error the error on the mean for signals at each bank angle.
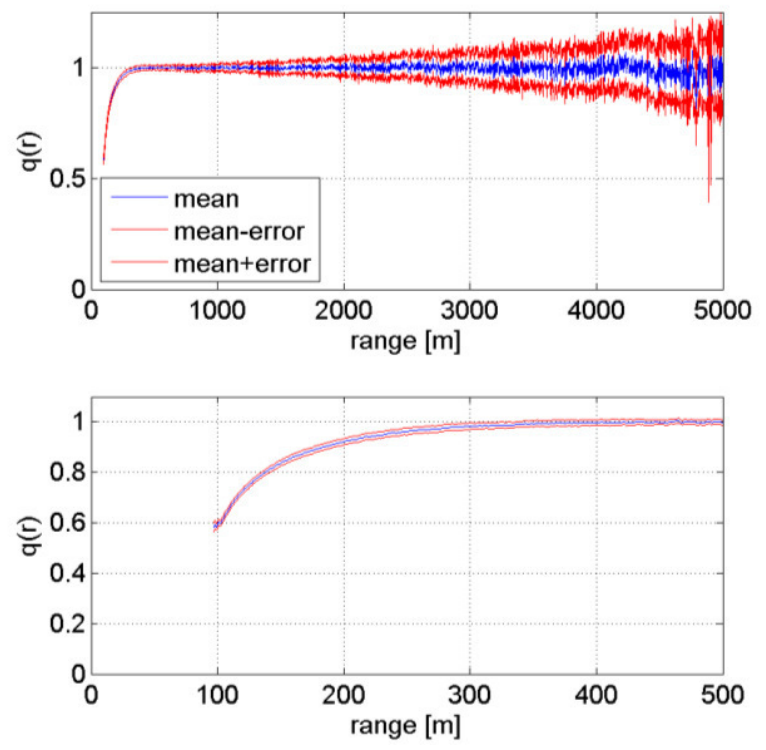

Fig. 6. Overlap function. The lower plot is zoomed region over $500 \mathrm{~m}$.

Note that if we consider from the beginning that signals from the first $300 \mathrm{~m}$ should not be considered, we arrive to the situation in which it is not possible to estimate the overlap function very close to the lidar. The highest the bank angles the closest to the lidar the overlap function can be retrieved. For more details please see [4], where the smallest the elevation angles the closest to the lidar the overlap function goes. Thus, technically, for $60^{\circ}$ bank angle, $300 \mathrm{~m}$ range corresponds to $150 \mathrm{~m}$ on altitude. The linear regression is performed if there are at least three points available (corresponding to three different bank angles). Thus, for the highest bank angles $\left(40^{\circ}\right.$, $50^{\circ}$ and $60^{\circ}$ ) the corresponding heights for a range of $300 \mathrm{~m}$ are $229.81 \mathrm{~m}, 192.84 \mathrm{~m}$ and $150 \mathrm{~m}$. Therefore the linear regression can be first performed at $229.81 \mathrm{~m}$ away from the lidar.
As seen in Fig. 5 a) the optical depth is slightly larger than the molecular optical depth and thus, the comparison of the measured RCS with synthetic molecular RCS is not appropriate.

A thorough presentation of the method and a complete picture of various steps will be shown during ILRC.

\section{CONCLUSIONS}

We have demonstrated an innovative method to determine the overlap function for an airborne Lidar. The main constraint in retrieving the overlap function very close to the Lidar is the availability of the measurements taken at high bank angles, which is possible up to an aircraftdependent limit (60 degrees for the BAe-146).

For the system on-board the FAAM aircraft, in the present study we estimate full overlap to be reached at $\sim 400 \mathrm{~m}$. This has to be compared with a nominal overlap of $300 \mathrm{~m}$; note that at this range we estimate the overlap function to be equal to 0.98 The overlap function was retrieved down to $\sim 95 \mathrm{~m}$ from the Lidar.

\section{ACKNOWLEDGEMENT}

Airborne data was obtained using the BAe-146 Atmospheric Research Aircraft flown by Directflight Ltd. and managed by the Facility for Airborne Atmospheric Measurements (FAAM), which a joint entity of the Natural Environment Research Council (NERC) and the Met Office.

\section{REFERENCES}

\section{[1] http://www.faam.ac.uk/}

[2] Marenco, F., J. Kent, M. Adam, J. Buxmann, P. Francis and J. Haywood, 2015, Remote Sensing of Volcanic Ash at the Met Office, 27 ILRC Hazard Monitoring.

[3] Marenco, F., et al., 2011: Airborne lidar observations of the 2010 Eyjafjallajökull volcanic ash plume, J. Geophys. Res., 116, D00U05, doi:10.1029/2011JD016396.

[4] Adam, M., et al., 2007: Application of the Kano-Hamilton Multiangle Inversion Method in Clear Atmospheres, J. Atmos. Oceanic Technol., 24, 2014-2028. 